\title{
Vaginal Ozone Insufflation in the Treatment of Recurrent Candidal Vulvovaginitis : Randomized Control Trial \\ Original
} Article

\author{
Hassan Khairy, Mohamed Ibrahim, Rasha Abdul Hadi, Hossam El-Taweel
}

Department of Obstetrics and Gynecology, Faculty of Medicine, Ain-Shams University, Cairo

\begin{abstract}
Background: O3 therapy induces moderate oxidative stress when interacting with lipids.

Aim: This work aimed to demonstrate the efficacy of the application of vaginal ozone insufflations in treatment of recurrent candidal vulvovaginitis compared to combined vaginal and systemic antifungal agents.

Materials and Methods: This randomized controlled study was conducted on 50 women with recurrent vulvovaginitis recruited from the outpatient gynecology clinic of Ain-shams University Obstetrics and Gynecology Hospital who were attending for outpatient consultation. Subjects were assigned randomly into two groups; group A included 25 subjects who were treated with traditional, combined, topical and systemic antifungal therapy and group B included 25 subjects who were treated by vaginal ozone insufflations.

Results: Twenty-two (88\%) patients in the vaginal ozone group had clinical cure compared with only 14 (56\%) patients in the traditional treatment group. This difference was statistically significant. Likewise, the microbiological cure rate was significantly higher in the vaginal ozone group compared with the traditional treatment group. Five out of 14 patients (35.7\%) in the traditional treatment group suffered clinical recurrence compared with only 1 out of 22 patients $(4.5 \%)$ in the vaginal ozone group. This difference was statistically significant. Likewise, the microbiological recurrence rate was significantly lower in the vaginal ozone group compared with the traditional treatment group.

Conclusion: Ozone therapy has proven to be effective in treatment of the recurrent vulvovaginitis, where the persistence of clinical symptoms and positive culture exudates were measured.
\end{abstract}

Key Words: Recurrent candidal vulvovaginitis, treatment, vaginal ozone insufflation

Received: 29 September 2019, Accepted: 29 September 2019

Corresponding Author: Hassan Khairy, Department of Obstetrics and Gynecology, Faculty of Medicine, Ain-Shams University, Cairo Tel.: 01069491030,E-mail: hassankhairy308@gmail.com

ISSN: 2090-7265, May 2021, Vol.11, No. 2

\section{INTRODUCTION}

Vaginitis caused by Candida albicans is the most common gynecological disease found in the primary health care and makes a health issue of great importance because of its high frequency ${ }^{[1]}$.

Vaginal yeast infection, also known as candidal vulvovaginitis and vagina] thrush, is excessive growth of yeast in the vagina that results in irritation. The most common symptom is vaginal itching, which may be severe. Other symptoms include burning with urination, white and thick vaginal discharge that typically does not smell bad, pain with sex, and redness around the vagina. Symptoms often worsen just before a woman's period ${ }^{[2]}$.

Vaginal yeast infections are due to excessive growth of Candida. These yeasts are normally present in the vagina in small numbers. It is not classified as a sexually transmitted infection; however, it may occur more often in those who are frequently sexually active. Risk factors include taking antibiotics, pregnancy, diabetes, and HIV/AIDS. Eating a diet high in simple sugar may also play a role. Tight clothing, type of underwear, and personal hygiene do not appear to be factors. Diagnosis is by testing a sample of vaginal discharge. As symptoms are similar to that of the sexually transmitted infections, chlamydia and gonorrhea, testing may be recommended ${ }^{[3]}$.

Despite the lack of evidence, wearing cotton underwear and loose fitting clothing is often recommended as a preventative measure. Avoiding douching and scented hygiene products is also recommended. Treatment is with an antifungal medication. This may be either as a cream such as clotrimazole or with oral medications such as fluconazole. Probiotics have not been found to be useful for active infections ${ }^{[4]}$.

About $75 \%$ of women have at least one vaginal yeast infection at some point in their lives while nearly half have at least two. About 5\% have more than three infections in a single year. It is the second most common cause of vaginal inflammation after bacterial vaginosis ${ }^{[5]}$.

A vulvo-vaginitis is considered recurrent when the event repeats four or more times per year. In that case, to carry out an adequate diagnosis and the ensuing treatment it is necessary 
to perfectly individualize each case, as noncomplicated candidiasis perfectly responds to common treatments, including those using single doses, while the complicated cases do not generally respond to single doses and require lengthy treatment periods or prophylactics (prophylaxis of the recurring infection $)^{[6]}$.

The conditions it might help include thrush, for which it is outstandingly effective, often where all else has failed. Ozone therapy involves the patient, not the practitioner, inserting a small catheter as far into the area as is comfortable and guiding ozone into it at a low flow rate. This method is painless, not at all intrusive, and does not cause discomfort of any kind for the patient. In fact, the practitioner does not need to stay in the room while the insufflations take place. The yeasts and microorganisms tend to be the basis of vaginal health problems, but are very sensitive to ozone. It's interesting how healthy body cells are not affected by ozone at all ${ }^{[7]}$.

Ozone treatment usually leads to a dramatic improvement in vagina symptoms, and it appears that the benefits continue for a considerable amount of time. It is a safe and non-toxic treatment, and can simply be repeated at appropriate time intervals $^{[7]}$

Vaginal ozone can be very promising in the treatment of recurrence vulvo-vaginitis, which is a long-term, chronic problem, which leads to psychological implication ${ }^{[4]}$.

\section{AIM OF THE WORK}

The aim of this study is to demonstrate the efficacy of the application of vaginal ozone insufflations in treatment of recurrent vulvo-vaginitis caused by Candida albicans species, compared to combined treatment with vaginal and systemic antifungal agents.

\section{PATIENTS AND METHODS}

The current randomized-controlled study included 50 women with recurrent vulvo-vaginitis, recruited from the outpatient gynecology clinic of Ain-Shams University Obstetrics and Gynecology Hospital, who were attending for outpatient consultation.

The study included subjects between 20 and 40 years of age and subjects who were clinically diagnosed with recurrent vulvo-vaginitis (four or more Candida vaginal infections during one year) with at least 6 months of refractory evolution to common pharmacologic treatment and positive cultures of candida albicans ${ }^{[8]}$.

Subject having non-willingness to participate in the study, pregnancy and lactation, presence of other vaginal infections such as bacterial vaginosis, use of fungicides or other locally applied medicines during the 2 weeks prior to the start of the treatment of the study, open wound in the reproductive system and patients with medical disorders such as (DM, and autoimmunie diseases who receive steroid therapy) were excluded from the study.

Careful and detailed history was acquired from all participants including; Personal history: name, age, occupation, residence, special habits of medical importance. Gynecological history especially, first day of last menstrual period. Past history: history of any medical disorder or surgical history Laboratory investigations: $\beta$-HCG titre and $\mathrm{CBC}$.

Grouping and assignment: subjects were assigned into two groups as follows ; group A included 25 subjects who were treated with traditional, combined, topical and systemic antifungal therapy, according to the following treatment regimen $/$ protocol ${ }^{[8]}$. Topical treatment; terconazole $80 \mathrm{mg}$ vaginal suppositories, subjects were instructed to use on daily basis for three successive days.

Systemic treatment: Stage of induction fluconazol capsule $(150 \mathrm{mg})$, every 72 hours (three days) for 3 doses. Stage of maintenance; fluconazol capsule $(150 \mathrm{mg})$, once per week for 6 months ${ }^{[8]}$. Group B included 25 subjects who were treated by vaginal ozone insufflations In both groups, the patients' partners received oral antifungal treatment (fluconazol capsule $(150 \mathrm{mg}$ ) one single dose).

Ozone treatment/therapy; vaginal insufflations were performed by insufflations of ozone/oxygen gas mix into the female reproductive system with the help of a catheter. They were performed in a continuous fashion, via attaching a catheter to the ozone output hose and allowing the ozone/ oxygen gas mix to flow-in for the desired amount of time. Equipment used in order to do vaginal insufflations was used ozone generator (Ozonosan ${ }^{\circledR}$ ozone generator (CDr. Hansler, Ozonosan, Iffezheim, Germany), (gauge between 20 and $40 \mu \mathrm{g} / \mathrm{ml}$ ) at oxygen flows of $1 / 32$ to $1 / 8$ liters per minute and the catheter (Nelaton catheter, size 18). Regimen/ technique/protocol: insufflations of medical ozone gas started at a low rate and a concentration of $20-40 \mu \mathrm{g} / \mathrm{ml}$, with a continuous flow of $50 \mathrm{ml} /$ minute, for 6 minutes ( 300 $\mathrm{ml}$ total volume), for every other day for $12 \operatorname{sessions}^{[7]}$. Bocci $^{[7]}$ study was adopted in the use of vaginal ozone insufflation for 6 minutes, which was more comfortable and easier for the patients, although different studies used for a longer period of time. An intravaginal silicone catheter, that was specially designed for this purpose was used, in order to allow optimal intravaginal ozonation, ensuring no leak of ozone, in addition to a more uniform contact of ozone on the vaginal epithelium.

Clinical and microbiological criteria for efficacy Complete clinical and microbiological healing Absence of subjective clinical symptoms Absence of inflammatory disorders of the vaginal mucous membrane, and negative vaginal cultures taken after ending the treatment. 
Follow up; it was considered that the subject has improved when a substantial reduction of the objective and subjective symptoms was evidenced. All subjects were followed up to detect relapse within the following three months by clinical symptoms. The infection was considered recurrent when the presence of subjective and/or objective clinical symptoms of candidiasis were detected with a positive vaginal culture or exudate after 2-4 weeks of having ended the treatment.

General clinical protocol: vaginal cultures and exudates were taken before and at the end of treatment and sent to the clinical laboratory for analysis.

\section{STATISTICAL ANALYSIS:}

Data were analyzed using IBM $\odot$ SPSS $\odot$ Statistics version 23 (IBMC Corp., Armonk, NY, USA). Continuous numerical variables were presented as mean and standard deviation (SD) and intergroup differences were compared using the independent-samples t-test. Categorical data were presented as number and percentage and differences were compared using Fisher's exact test. The absolute risk reduction (ARR) and number needed to treat (NNT) were used to assess the therapeutic efficacy of vaginal ozone for eradication of cadididal vulvovaginitis. The NNT is the reciprocal of the ARR $(\mathrm{NNT}=1 / \mathrm{ARR}) . P$-values $<0.05$ were considered statistically significant.

\section{RESULTS}

Table 1 showed the demographic characteristics of both study groups. There is no statistically significant difference between both groups as regards the age $(P$-value $=0.366)$, BMI $(P$-value $=0.454)$ or parity $(P$-value $=0.773)$.

In the traditional treatment group, $17(68 \%)$ patients suffered from 3-6 episodes of vulvovaginitis per year, while $8(32 \%)$ had $>6$ episodes per year. In the vaginal ozone group, $16(64 \%)$ patients had 3-6 episodes per year and $9(36 \%)$ patients had $>6$ episodes per year. This difference was not statistically significant $(P$-value $=1.000)$.

Table 2 showed the clinical and microbiological cure rates in both study groups. Twenty-two (88\%) patients in the vaginal ozone group had clinical cure compared with only $14(56 \%)$ patients in the traditional treatment group. This difference was statistically significant $(P$-value $=0.025)$.

Likewise, the microbiological cure rate was significantly higher in the vaginal ozone group compared with the traditional treatment group ( $88 \%$ versus $52 \%$, respectively, $P$-value $=0.012$ ).

Table 3 showed the results of risk analysis for failure to achieve clinical or microbiological cure. The risk of failure to achieve clinical cure was significantly lower for the vaginal ozone group with a relative risk (risk ratio, RR) of $0.27(95 \% \mathrm{CI}=0.09$ to 0.86$)$, absolute risk reduction of $0.32(95 \% \mathrm{CI}=0.09$ to 0.55$)$ and number needed to treat $(\mathrm{NNT})$ of $3.13(95 \% \mathrm{CI}=11.44$ to 1.81$)$.

Similarly, the risk of failure to achieve microbiological cure was significantly lower for the vaginal ozone group with a relative risk (risk ratio, RR) of $0.25(95 \% \mathrm{CI}=0.08$ to 0.78$)$, absolute risk reduction of $0.36(95 \% \mathrm{CI}=0.13$ to 0.59$)$ and number needed to treat (NNT) of $2.78(95 \%$ $\mathrm{CI}=7.91$ to 1.68 ).

Table 4 showed the clinical and microbiological recurrence rates in both study groups. Five out of 14 patients (35.7\%) in the traditional treatment group suffered clinical recurrence compared with only 1 out of 22 patients $(4.5 \%)$ in the vaginal ozone group. This difference was statistically significant $(P$-value $=0.024)$.

Likewise, the microbiological recurrence rate was significantly lower in the vaginal ozone group compared with the traditional treatment group (4.5\% versus $38.5 \%$, respectively, $P$-value $=0.019$ ).

Table 5 showed the results of risk analysis for clinical or microbiological recurrence. The risk of clinical recurrence was significantly lower for the vaginal ozone group with a relative risk (risk ratio, RR) of $0.13(95 \% \mathrm{CI}=0.02$ to 0.98$)$, absolute risk reduction of $0.31(95 \% \mathrm{CI}=0.05$ to 0.58$)$ and number needed to treat (NNT) of $3.21(95 \%$ $\mathrm{CI}=21.73$ to 1.73 ).

Similarly, microbiological recurrence was significantly lower for the vaginal ozone group with a relative risk (risk ratio, $\mathrm{RR})$ of $0.12(95 \% \mathrm{CI}=0.02$ to 0.90$)$, absolute risk reduction of $0.34(95 \% \mathrm{CI}=0.06$ to 0.62$)$ and number needed to treat $(\mathrm{NNT})$ of $2.95(95 \% \mathrm{CI}=16.46$ to 1.62$)$. 
Table 1: Demographic characteristics of both study groups

\begin{tabular}{lcccc}
\hline Variable & $\begin{array}{c}\text { Traditional treatment } \\
\text { group }(\mathrm{n}=25)\end{array}$ & $\begin{array}{c}\text { Vaginal ozone } \\
\text { group }(\mathrm{n}=25)\end{array}$ & Difference & 95\% CI \\
\hline Age (years) & $30.3 \pm 5.6$ & $31.7 \pm 4.9$ & 1.4 & -1.68 to 4.48 \\
BMI (kg/m2) & $25.8 \pm 4.3$ & $26.0 \pm 3.1$ & -0.8 & -2.93 to 1.33 \\
Parity & $11(44 \%)$ & $9(36 \%)$ & - & - \\
Primipara & $14(56 \%)$ & $16(64 \%)$ & & 0.754 \\
Multipara & & & & - \\
Frequency of vulvovaginitis & $17(68 \%)$ & $16(64 \%)$ & & - \\
3-6 episodes/year & $8(32 \%)$ & $9(36 \%)$ & \\
$>6$ episodes/year & & & \\
\hline
\end{tabular}

Table 2: Clinical and microbiological cure rates in both study groups

\begin{tabular}{|c|c|c|c|}
\hline Variable & $\begin{array}{l}\text { Traditional treatment } \\
\text { group }(n=25)\end{array}$ & Vaginal ozone group $(n=25)$ & $P$-value* \\
\hline Clinical cure & $14(56 \%)$ & $22(88 \%)$ & 0.025 \\
\hline Microbiological cure & $13(52 \%)$ & $22(88 \%)$ & 0.012 \\
\hline
\end{tabular}

Table 3: Risk analysis and number needed to treat for clinical or microbiological cure

\begin{tabular}{|c|c|c|c|c|c|c|}
\hline & \multicolumn{6}{|c|}{ Outcome measure } \\
\hline & \multicolumn{2}{|c|}{ Clinical cure } & & \multicolumn{3}{|c|}{ Microbiological cure } \\
\hline N. cured in ozone group & \multicolumn{2}{|c|}{22} & & \multicolumn{3}{|c|}{22} \\
\hline $\begin{array}{l}\text { N. with persistent infection } \\
\text { in ozone group }\end{array}$ & \multicolumn{2}{|c|}{3} & & \multicolumn{3}{|c|}{3} \\
\hline N. cured in traditional group & \multicolumn{2}{|c|}{14} & & \multicolumn{3}{|c|}{13} \\
\hline $\begin{array}{l}\text { N. with persistent infection } \\
\text { in traditional group }\end{array}$ & \multicolumn{2}{|c|}{11} & & \multicolumn{3}{|c|}{12} \\
\hline Risk estimate & Value & $95 \% \mathrm{LCL}$ & $\begin{array}{l}95 \% \\
\text { UCL }\end{array}$ & Value & $95 \% \mathrm{LCL}$ & $95 \% \mathrm{UCL}$ \\
\hline AR in treatment group & 0.12 & -0.01 & 0.25 & 0.12 & -0.01 & 0.25 \\
\hline AR in control group & 0.44 & 0.25 & 0.63 & 0.48 & 0.28 & 0.68 \\
\hline ARR & 0.32 & 0.09 & 0.55 & 0.36 & 0.13 & 0.59 \\
\hline $\mathrm{RR}$ & 0.27 & 0.09 & 0.86 & 0.25 & 0.08 & 0.78 \\
\hline RRR & 0.73 & 0.14 & 0.91 & 0.75 & 0.22 & 0.92 \\
\hline Odds in treatment group & 0.14 & & & 0.14 & & \\
\hline Odds in control group & 0.79 & & & 0.92 & & \\
\hline OR & 0.17 & 0.04 & 0.73 & 0.15 & 0.04 & 0.62 \\
\hline NNT & 3.13 & 11.44 & 1.81 & 2.78 & 7.91 & 1.68 \\
\hline
\end{tabular}

Data in cross-tables are counts.

N. $=$ number, 95\% LCL $=95 \%$ lower confidence limit, 95\% UCL $=95 \%$ upper confidence limit, $\mathrm{AR}=$ absolute risk, $\mathrm{ARR}=$ absolute risk reduction, $\mathrm{RR}=$ relative risk, $\mathrm{RRR}=$ relative risk reduction, $\mathrm{OR}=$ odds ratio, $\mathrm{NNT}=$ number needed to treat. 
Table 4: Clinical and microbiological recurrence rates in both study groups

\begin{tabular}{lccc}
\hline Variable & Traditional treatment group & Vaginal ozone group & $P$-value* \\
\hline Clinical recurrence & $5 / 14(35.7 \%)$ & $1 / 22(4.5 \%)$ & 0.024 \\
Microbiological recurrence & $5 / 13(38.5 \%)$ & $1 / 22(4.5 \%)$ & 0.019 \\
\hline
\end{tabular}

Data are proportion (\%).

*Fisher's exact test.

Table 5: Risk analysis and number needed to treat for clinical or microbiological recurrence

Outcome measure

N. with persistent cure

in ozone group

$\mathrm{N}$. with recurrence

in ozone group

N. with persistent cure

in traditional group

$\mathrm{N}$. with recurrence in

traditional group

Risk estimate

$\mathrm{AR}$ in treatment group

AR in control group

ARR

RR

RRR

Odds in treatment group

Odds in control group

OR

NNT
Value

0.05

0.36

0.31

0.13

0.87

0.05

0.56

0.09

3.21
21

Data in cross-tables are counts.

95\% LCL $=95 \%$ lower confidence limit, 95\% UCL $=95 \%$ upper confidence limit, $\mathrm{AR}=$ absolute risk, $\mathrm{ARR}=$ absolute risk reduction, $\mathrm{RR}=$ relative risk, $\mathrm{RRR}=$ relative risk reduction, $\mathrm{OR}=$ odds ratio, $\mathrm{NNT}=$ number needed to treat. 


\section{DISCUSSION}

Ozone trioxygen $\left(\mathrm{O}_{3}\right)$ is a highly reactive inorganic molecule. It is a natural part of the atmosphere also produced by ozone generators. Although ozone was discovered in the mid-nineteenth century, its medicinal usage was discovered during the recent decades. It was found that ozone is one of the best microorganism killers. As such, it has antibacterial, antiviral, antifungal, and anti-parasitic properties. In addition, ozonated oils (oleozone) are used in the medical and pharmaceutical products and in textile and food industries ${ }^{[9]}$.

The current study objective was to demonstrate the efficacy of the application of vaginal ozone insufflations in treatment of recurrent vulvo-vaginitis caused by Candida albicans species compared to combined treatment with vaginal and systemic antifungal agents.

The study was designed to be a randomized controlled study; it was conducted at Ain-Shams University Maternity Hospital. The study included 50 women with recurrent vulvo-vaginitis, recruited from the outpatient gynecology clinic of Ain-Shams University Obstetrics and Gynecology Hospital who were attending for outpatient consultation.

Subjects were subcategorized into two major groups, each comprised of 25 subjects; group A subjects were treated with traditional systemic and local antifungal therapy and group $\mathrm{O}$ who were treated using ozone insufflations.

There was a statistically significant difference between both groups as regards laboratory investigations done (high vaginal swap or virginal exudates culture and sensitivity) as well as the clinical subjective symptoms (e.g. itching, excoriations, redness ... etc), by default, all of the pretreatment specimens were all positive; nevertheless, the post treatment specimens. However, no statistically difference was found between the studied groups as regards the demographic data (age, body mass index, parity ... etc.).

The total number for the persistence of the clinical symptoms as well as the positivity of the culture did indeed differ significantly, 16 subjects from group A were refractory to treatment against 4 subjects from group $\mathrm{O}$.

Ozone is a chemical compound composed of three oxygen atoms that has higher energy content than atmospheric oxygen. This compound has medicinal (for disinfecting and treating microbial infections and non-microbial diseases), industrial, and agricultural applications. Ozone is used in the treatment of recurrent vulvovaginal candidiasis, and it has synergistic effect with chlorhexidine for the treatment of infected root canals ${ }^{[10]}$.

In a study by Zargaran et al. ${ }^{[10]}$ ozone was highly effective in killing C. albicans in yeast form and inhibition of germ tube formation during 210 and 180 s, respectively.
Although with increasing exposure time biofilm production was considerably decreased, resistance to ozone was much higher among vaginal and nail isolates even after $60 \mathrm{~min}$. All the strains were sensitive to fluconazole, caspofungin, and terbinafine pre- and post-ozone exposure. Resistance to amphotericin B was significantly enhanced after exposure to ozone.

In a study by Restaino et al., the inactivation of a 104-106 CFU/ml suspension of C. albicans was observed after 5 min of ozone exposure. da Silva Faria et al. believed that clinical strains of Candida were more resistant to ozone than non-clinical isolates. They found that ozone exposure for $5 \mathrm{~min}$ was not fully effective in the inactivation of these types of isolates. Furthermore, in a study by Cardoso et al., the complete inactivation of C. albicans was observed after $40 \mathrm{~min}$ of exposure to ozonated water with $600 \mathrm{ppm}$ ozone.

On the other hand, in brushes bristles exposed to ozonated PBS, complete sanitation was observed after $30 \mathrm{~min}$ (Cardoso et al. 2008, Bezirtzoglou et al. 2008, da Silva Faria et al., 2015, Restaino et al. 2015).

Moreover, in another study by Zargaran et al ${ }^{[10]}$ it was indicated that the most resistant isolates to ozone were those of the oral cavity and vulvovaginal candidiasis (same effect). Whereas urine isolates of C. albicans were more sensitive to ozone than the other isolates, such that after 150 s of exposure, they were fully inhibited by ozone. It seems that other factors such as the initial suspension concentration and sources of isolates could affect fungicidal activity of ozone.

Germ tube formation in C. albicans is the first step in adhesion to host tissues and acrylic surfaces; therefore, it is considered as one of the important pathogenic factors. In a study by Ali et al. ${ }^{[11]}$ on diabetic foot ulcer isolates, germ tube formation in C. albicans was completely inhibited when exposed to $3 \mathrm{ppm}$ for $180 \mathrm{~min}$. In contrast, in another study, germ tube inhibition started at 60s exposure and completely stopped at $180 \mathrm{~s}^{[10]}$.

This is in agreement with our represented data, as in our study, ozone definitely eradicated C. albicans more than the traditional treatment.

Six-minute time for 12 sessions was adopted by Bocci et $a l^{[12]}$ in vaginal ozone insufflation which was more comfortable and easier for the patients, although other studies applied a longer period of time.

The biofilms of $\mathrm{C}$. albicans are resistant to several antifungals and their elimination plays a major role in the treatment of the disease. The previous studies have shown that ozone is a suitable tool for the removal of biofilm from dentures. Therefore, considering the formation of biofilms, especially in catheters, urethral catheters, and dentures, and their resistance to antifungals, it can be stated that ozone gas can help eliminate biofilms ${ }^{[13]}$ 
Interestingly, in another study, biofilms produced by vaginal and nail isolates showed greater resistance to ozone than the other isolates, which is in accordance with its yeast form resistance. In a study, $30 \mathrm{~min}$ exposure of acrylic dentures to high concentrations of ozonated water at $60^{\circ} \mathrm{C}$ completely removed Candida biofilms ${ }^{[14]}$.

In another study on yeast biofilm, it was found that the disinfecting action of ozone was less effective than the yeast form. In another study, vaginal and nail isolates were resistant to ozone even after $60 \mathrm{~min}$ of exposure, which was confirmed by Muller et al., who believed that ozone was not able to completely eliminate biofilms ${ }^{[15]}$.

This is in agreement with our reported data, as in our methodology, vaginal ozone insufflations were done every other day for 12 sessions, which may have contributed to yielding such a high rate of eradication.

\section{CONCLUSION}

To be concluded, ozone therapy is still juvenile and needs further investigations in other fields of infection treatment. Moreover, more studies including much population number of subjects are needed to determine the frequency of treatment and the duration.

Our vision is to use vaginal ozone insufflation in other types of vaginal infection as anaerobic bacterial vaginosis.

Ozone therapy has proven to be effective in treatment of the recurrent vulvovaginitis, where the persistence of clinical symptoms and positive culture exudates were measured.

\section{CONFLICT OF INTEREST}

There are no conflicts of interests.

\section{REFERENCES}

1. SchwartzA. (2015): Ozone therapy in the treatment of recurrent vulvo-vaginitis by Candida albicans. Revista Espaniola de Ozonoterapia, 5(1):99-107.

2. Krapf JM. (2017): Vulvovaginitis. Medscape.

3. Sobel JD. Vulvovaginal candidosis. Lancet, 2017; 369(9577): 1961-71.

4. Abad CL and Safdar N. The role of lactobacillus probiotics in the treatment or prevention of urogenital infections-a systematic review. J Chemother., 2016; 21 (3):243-52.

5. Ilkit M, Guzel AB. (2016): The epidemiology, pathogenesis, and diagnosis of vulvovaginal candidosis: a mycological perspective. Crit Rev Microbiol., 37(3): 250-61.

6. Nogales CG et al. (2014): Comparison of the antimicrobial activity of three different concentrations of aqueous ozone on Pseudomonas aeruginosa, Staphylococcus aureus, and Enterococcus faecalis - in vitro study. Revista Espaniola de Ozonoterapia, 4(1):9-15.

7. Bocci V. Ozone: A new medical drug. Springer, Netherlands. pp. 295. Springer; 1st Edition. 2010. ISBN-10: 9048168058.

8. Hoffman BL. (2016): Gynecologic Infection. In: Hoffman BL, Schorge JO, Bradshaw KD, Halvorson LM, Schaffer JI, Corton MM (Editors), William's Gynecology, 3rd Edition. Section 1; Chapter 3. McGraw Hill Education Inc. P. 50. 2016.

9. Zanardi I, Burgassi S, Paccagnini E, Gentile M, Bocci V, Travagli V. What is the best strategy for enhancing the effects of topically applied ozonated oils in cutaneous infections? Biomed Res Int 2013; 2013:702949.

10. Zargaran M, Fatahinia M, Zarei Mahmoudabadi A. The efficacy of gaseous ozone against different forms of Candida albicans. Curr Med Mycol. 2017; 3(2): 26-32.

11. Ali EM. Ozone application for preventing fungal infection in diabetic foot ulcers. Diabetol Croatica. 2013; 42(1):3-22.

12. Bocci VA, Zanardi I, Travagli V. Ozone acting on human blood yields a hormetic dose-response relationship. J Transl Med. 2011 (a);9:66.

13. Mahmoudabadi ZA, Zarrin M, Kiasat N. Biofilm formation and susceptibility to amphotericin B and fluconazole in Candida albicans. Jundishapur J Microbiol. 2014; 7(7):e17105.

14. Wiench R, Ilków A, Dziedzic A, Wojtyczka R, Cieślikwegemund $\mathrm{M}$, Kubicka-musiał $\mathrm{M}$, et al. The eradication of Candida spp. from removable dentures exposed to ozonated water. J Clin Periodontol. 2015; 42:109.

15. Mohammadi Z, Soltani MK, Shalavi S. An update on the management of endodontic biofilms using root canal irrigants and medicaments. Iran Endod J. 2014; 9(2):89-97. 